\title{
HOW TO ELIMINATE NON-DAMAGING EARTHQUAKES FROM THE RESULTS OF A PROBABILISTIC SEISMIC HAZARD ANALYSIS (PSHA) - A COMPREHENSIVE PROCEDURE WITH SITE SPECIFIC APPLICATION
}

\author{
Dr. Jens-Uwe Klügel \\ NPP Goesgen-Daeniken CH- 4658 Daeniken, ikluegel@kkg.ch
}

Abstract

From 2000 to 2004 the Swiss nuclear power plants sponsored a large scale probabilistic seismic hazard analysis following the SSHAC-procedures at its most elaborated level 4. This research project was intended to be used as a support of the update of the Goesgen plant-specific PSA. A detailed review based on the development of site-specific controlling earthquakes indicated that the obtained results included the effects of non-damaging earthquakes, which may potentially cause significant accelerations at the plant site not exceeding the acknowledged limits of an operational earthquake as defined in NRC RG 1.166 by a threshold value of 0.16 gs for the cumulative absolute velocity (CAV). Therefore, a detailed procedure was developed to eliminate non-damaging earthquakes from the results of a SSHAClevel 4 PSHA. The procedure allows to calculate the probability of exceedance of the damaging threshold of an operational earthquake. A key element of the procedure consists in the use of a correlated bivariate Monte Carlo bootstrap technique to address the epistemic uncertainties associated with the definition of scenario earthquakes and the use of multiple equations for the calculation of CAV. The implementation of the procedure in the site specific seismic PSA leads to a significant reduction of the calculated core damage frequency. It was also demonstrated that the deterministic design basis Safe Shutdown Earthquake (SSE) for Goesgen matches the theoretical Safe Shutdown Earthquake derived from PEGASOS after application of the procedure to a reasonable extent.

\section{INTRODUCTION}

From 2000 to 2004 the Swiss nuclear power plants sponsored a large scale probabilistic seismic hazard analysis following the SSHAC-procedures ([1]) at its most elaborated level 4. The project was requested by the Swiss Nuclear Safety Inspectorate based on recommendations and consultancy with US NRC and commercial American PSHAconsultants. This research project was intended to be used to support the update of the Goesgen plant-specific seismic PSA. The PEGASOS project led to somewhat surprising results (in comparison to empirical data from earthquake observations, [2]) indicating that the involved experts believe that the seismic activity in Switzerland for the next 30-40 years (the approximate residual lifetime of NPP Goesgen) is texpected to be similar to seismically more active regions like Turkey, Iran or even close to Japan and California. A detailed review of the underlying SSHAC procedures revealed [3],[4], [5] that these surprising results are directly caused by flaws in the SSHAC procedures. These meanwhile rather well-documented flaws are:

- The use of inconsistent modelling assumptions leading to an incorrect calculation of the probability of exceedance of earthquake ground motion levels (accelerations) [5].

- The violation of energy conservation principles caused by summing up frequency contributions to a certain acceleration level from weak (low energy) earthquakes and from strong earthquakes. Additionally, this summing neglects that these frequencies represent different statistical characteristics (fractiles) of the underlying probability distributions of ground motion exceedance. Therefore, this summing is mathematically very questionable. It cannot be proven that the associated sum of incoherent statistics (sampled from different distributions or more accurately, different stochastic processes of earthquake occurrence) converges to the true probability distribution of ground motion exceedance at a given site.

- The use of inadequate expert opinion elicitation and aggregation methods [3], [4], which are based on political consensus principles rather than on principles of rational consensus [6], [7].

The lack of consideration of energy conservation principles has the consequence that the output of a PSHA - the hazard curves or the so-called uniform hazard spectrum - is driven by low energy near-site earthquakes. Such earthquakes do not even cause damage at very high accelerations as has been shown by an increasing number of observations of high accelerations from small to moderate earthquakes near the sites of nuclear power plants. Vanmarcke and Lai [8] have shown that for a given content of elastic energy (expressed by the ARIAS intensity [9]) the strong motion duration reduces nearly proportionally with the increase of the peak values of peak ground acceleration.

The seeming contradiction between observed high accelerations and lack of damage - although such would have been predicted by current calculation procedures - can be explained by the prevailing use of linear-elastic calculation methods based on response spectra (for example, soil-structure interactions in industrial practice are calculated in the frequency 
domain, e.g. using the SASSI code). These methods are not capable to account for the difference in the energy content of low magnitude and high magnitude events correctly. Neglecting this problem in risk analysis would lead to a significant overestimation of seismic risk with a possible adverse impact on risk-informed decision making. This puts a strong challenge to risk analysts intending to develop a realistic assessment of seismic risk as it is necessary for responsible decision-making in an increasingly risk-informed environment. The most important correction to be made consists in the restoration of energy conservation principles with respect to the results of PSHA. There are two possible ways of restoring energy conservation principles in the aftermath of a PSHA:

1. Introduction of an energy threshold defining the onset of damage for well designed structures and components.

2. Scaling damage effects to energy measures and implementing correction factors into the fragility analysis.

The first approach leads to an incomplete restoration of energy conservation. Nevertheless, this approach may be sufficient to remove some of the most important risk contributors by damping the effects of small near site earthquakes.

The second approach is more promising [10] but not yet mature for application. The procedure presented here follows the first approach and is based on the implementation of an energy threshold expressed in terms of a critical CAV

(Cumulative Absolute Velocity) value of 0.16gs [11].

\section{GENERAL DESCRIPTION OF THE PROCEDURE FOR THE ELIMINATION OF NON-DAMAGING EARTHQUAKES}

\subsection{Short description of the PEGASOS project}

The PEGASOS project following the SSHAC procedures ([1] consisted of 4 separated subprojects:

- SP1 "Source Characterisation” with four different expert groups

- SP2 “Ground Motion Characteristics" developing the hazard on generic rock - 5 experts

- SP3 "Site Response Characterisation” propagating the hazard from generic rock to site surface and different subsurface layers -4 experts

- SP4 "Hazard Quantification" - performing computational tasks. Hazard quantification was performed with FRISK88M (discrete probability approach, ([12]).

The results were presented in the format of seismic hazard curves and uniform hazard spectra.

\subsection{Description of the procedure}

The procedure for the elimination of non-damaging earthquakes consists of the following steps:

1. Disaggregation of the hazard in terms of probability of exceedance for different distance ranges, different spectral frequencies and for each of the single expert opinion combinations taking different disaggregation procedures into account (US NRC RG 1.165 [13] and Bazzuro and Cornell,[14]). This allows to develop an epistemic uncertainty distribution of controlling events for each of the considered distance ranges (in this case D1<16km, D2 16-40km, D3 $>40 \mathrm{~km}$ ). The results were presented in terms of magnitude and distance pairs, representing scenario earthquakes. The resulting set of scenario events (typically about 120 magnitude-distance pairs for each probability of exceedance for one distance range) were interpreted as the discrete bivariate epistemic probability distribution of controlling (scenario) seismic events.

2. Selection/development of site-specific empirical correlations for the cumulative absolute velocity CAV. Analysis of influencing model parameters (e.g. focal depth) and assessment of epistemic uncertainty. According to NRC RG 1.166 [11] and the underlying supporting investigations the damaging threshold of an earthquake is defined by a CAV threshold value of 0.16 gs. Empirical correlations have to be developed by nonlinear regression. The regression residuals are interpreted as spatial randomness of earthquake time histories at different locations (combines interevent and intra-event variability).

3. Development of a Monte Carlo (Bootstrap) procedure for correlated sampling of controlling events (from the developed discrete bivariate distribution) and estimation of CAV-threshold exceedance.

4. Development of a composite epistemic uncertainty distribution for the exceedance probability of the CAV threshold value for the different distance ranges.

5. Development of a composite epistemic uncertainty distribution for the uniform hazard spectra (UHS).

6. Application of the composite distribution either for a direct adjustment of the hazard curves or in the PSA model or for the evaluation of the split fractions used to calculate the exceedance of the damaging threshold of earthquakes.

7. Development of an SSE compatible design spectrum for comparison with the existing design basis. 


\section{EMPIRICAL EQUATIONS FOR THE CALCULATION OF THE CUMULATIVE ABSOLUTE VELOCITY (CAV)}

The development of a model or of empirical equations for the calculation of the cumulative absolute velocity is a key step of the procedure for the elimination of non-damaging earthquakes. In the ideal case the same set of data shall be used for the development of an empirical equation for CAV as was used for the development of the attenuation model of the PSHA. In case of the PEGASOS project this was not feasible because the original data used for the development of the different attenuation models (a total of 15 models was considered by the experts) applied in the project were not retrieved from the authors of the different models. Therefore, it was necessary to perform a detailed estimation of epistemic uncertainties associated with the use of different models or different data sets.

\subsection{European equations for CAV}

Kostov [15] has derived a set of attenuation equations for CAV for different distance ranges, using the database of European earthquake time histories. At present, Kostov's work is the only publicly available information on the dependence of CAV on magnitude and distance for European conditions. Please note that Kostov was not able to provide a classification of the ground characteristics for the measurement points of the European earthquake recordings used.

The equation for near-site low to moderate magnitude earthquakes obtains the following form:

$$
\log (C A V)=-3.55+0.606 M_{s}-0.461 \log (R)+P \sigma
$$

with the distance measure $R$ based on the epicentral distance $D_{\text {epi }}$ and the focal depth $h$. $M_{S}$ stands for surface magnitude. The uncertainty is given as $\sigma=0.21$, but due to the need to extrapolate to larger magnitude values an additional epistemic component shall be considered. Therefore, a value of $\mathbf{0 . 2 5}$ is used (corresponds to the typical values for attenuation equations for spectral accelerations).

$$
R=\sqrt{\left(D_{e p i}^{2}\right)+h^{2}}
$$

A second equation was developed by Kostov to calculate CAV in dependence of magnitude and distance for the more distant regional sources. This equation can be used to evaluate the exceedance probability of the critical CAV value of 0.16 gs for the more distant seismic sources (for the associated controlling earthquakes):

$$
\log (C A V)=-2.88+0.44 M_{S}-0.565 \log (R)+\sigma P
$$

The associated value for $\sigma$ is higher than for equation (1), $\sigma=0.37$.

The epicentral distance can be calculated from the Joyner-Boore-Distance $R_{J B}$, used in the PEGASOS project from the following equation (Klügel, 2005):

$$
\begin{gathered}
D_{e p i}=R_{J B}+\varepsilon \\
\varepsilon=-0.86548+0.0206 M^{2.8861}
\end{gathered}
$$

$M$ here is the moment magnitude. The conversion of moment magnitude $M_{W}$ to surface wave magnitude $M_{S}$ is based on the equation of Ekström and Dziowonski [16].

To apply Kostov's equation to the PEGASOS results, the development of a statistical model for the distribution of focal depths in dependence of magnitude was required. Only the experts of SP1 provided some estimate for the focal depth of earthquakes in Switzerland, assuming a modal value of the focal depth distribution of $10 \mathrm{~km}$ based on observations of minor (low magnitude earthquakes). This information was used to develop a Gamma-distribution for focal depths with magnitude dependent mean and variation, considering the statistical dependence of focal depth on magnitude discussed by Toro [17] as part of the PEGASOS project. The obtained gamma-distribution was used to calculate a randomised set of focal depths, corresponding to the magnitude value of the randomly selected scenario earthquake.

\subsection{Development of site-specific empirical correlations for CAV from the WUS-database (EPRI 2005)}

Two different sets of empirical equations were developed from the published WUS (Western United States and all over the world) database (EPRI [18]). These published data sets are strongly classified with respect to the relevant ground 
characteristics in terms of the governing shear wave velocity $\left(V s_{30}\right)$. They contain a large amount of data from sites with similar values of shear wave velocity as the Gösgen site (in the range between $300 \mathrm{~m} / \mathrm{s}$ and $500 \mathrm{~m} / \mathrm{s}$ ). A total of 1541 datasets was found to match the range of shear wave velocities measured for the Goesgen site (Anatolian Geophysical, 2006).

The first set of equations was developed following the regression shape suggested by Kostow, replacing the focal depth $h$ by a free regression parameter, due to the lack of information on focal depths in the WUS database ([18]. The following regression shape was selected for the first correlation (WUS 1):

$$
\log (C A V)=b_{1}+b_{2} M+b_{3} \log (R)+b_{4} R+b_{5}(M-5.0)^{2}
$$

with the changed meaning of $h$ as stated above. A similar correlation was developed for pga using the same datasets. The obtained coefficients are presented in table 1.

Table 1. Regression coefficients and standard deviation for the correlation WUS $1(300 \mathrm{~m} / \mathrm{s}<$ Vs $<$ $500 \mathrm{~m} / \mathrm{s})$.

\begin{tabular}{|l|l|l|l|l|l|l|l|}
\hline Parameter & b1 & b2 & b3 & b4 & b5 & h & $\sigma$ \\
\hline $\log (\mathrm{CAV})$ & -5.9266 & 1.3811 & -2.0284 & 0.0036717 & -0.26281 & 17.952 & 0.37226 \\
\hline $\log (\mathrm{pga})$ & -3.2922 & 0.73851 & -1.4135 & 0.0036421 & -0.17693 & $11.94^{1}$ & 0.22051 \\
\hline
\end{tabular}

Figure 1 (left) shows the dependence of CAV on magnitude and distance according to the used data 2 and the residual plot for the obtained regression equation (right).
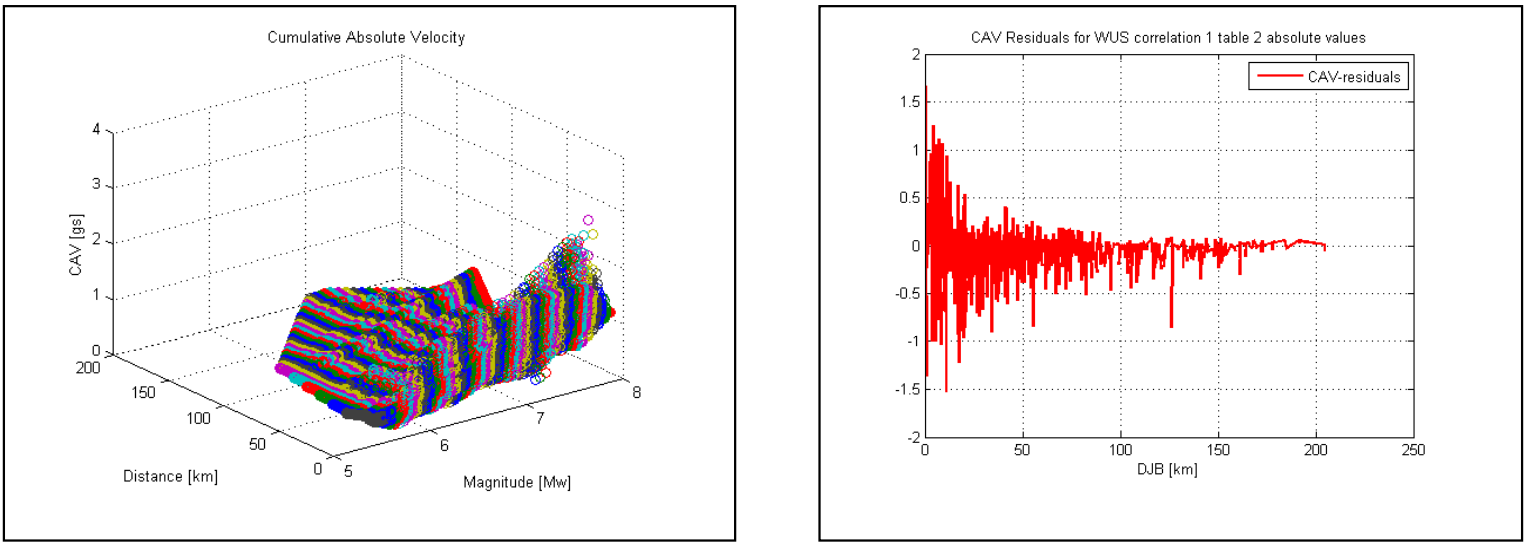

Figure 1. CAV in dependence on magnitude and distance and residual plot

A second interpretation of the data given in [18] and representative for Goesgen site conditions was developed following an idea of [18] using uniform duration [19] as a scaling parameter. A correlation between CAV, pga and the uniform duration of an earthquake can be suggested in analogy to [8], where a correlation between the strong motion duration, the pga and the Arias-intensity was established. In this analogy, the uniform duration can be considered as the equivalent of the strong motion duration. This analogy indicates that the following regression shapes appear to be meaningful:

$$
\begin{gathered}
C A V=a_{1} * p g a^{a_{2} *} d_{u}^{a_{3}}+a_{4} \\
\log \left(d_{u}\right)=c_{1}+c_{2} M+c_{3} \log (R)+c_{5} /(1+p g a)
\end{gathered}
$$

\footnotetext{
${ }^{1}$ The regression resulted in a negative value for the "artificial focal depth" - the solution for this parameter is not unique because of the square root of the sum of squares in the equation. The positive branch of the solution was selected.
} 
Here, $d_{u}$ is the uniform duration. Because the calculation of CAV is defined for time windows of a length of 1 s for a meaningful regression, it was necessary to consider only datasets with a uniform duration of a length of at least $1 \mathrm{~s}$ in the regression for the uniform duration. Nevertheless, 1064 datasets were retained for the regression analysis. Tables 2 and 3 show the coefficients for equations (10) and (11), obtained by nonlinear regression. The results of the regression confirm the almost linear dependency between CAV and the uniform duration for a fixed pga-value ( $a_{3}$ is close to 1 ). Equations (10) and (11) are abbreviated below as WUS correlation 2.

Table 2. Coefficients of the alternative regression equation (10) for CAV.

\begin{tabular}{|l|l|l|l|l|l|}
\hline Parameter & a1 & a2 & a3 & a4 & $\sigma$ \\
\hline CAV & 0.13435 & 0.34454 & 0.96193 & 0.042448 & 0.060404 \\
\hline
\end{tabular}

Table 3. Coefficients of the regression equation (11) for uniform duration.

\begin{tabular}{|l|l|l|l|l|l|l|}
\hline Parameter & c1 & c2 & c3 & c4 & c5 & $\sigma$ \\
\hline $\mathrm{d}_{\mathrm{u}}$ & -0.099174 & 0.32521 & -0.90306 & 14.992 & -0.014763 & 0.2217 \\
\hline
\end{tabular}

Figures 3 and 4 show a comparison of the different equations for the calculation of CAV. The comparison shows that some significant differences can be observed between the different equations. It is worth to mention that equations (12) and (13) will result in some overestimation of CAV because a larger amount of explanatory variables was used (and considered as uncorrelated in the regression). The multi-step regression procedure used increases the regression error and leads to a convolution effect (increased mean as a pay-off for reduced variability of the residuals).
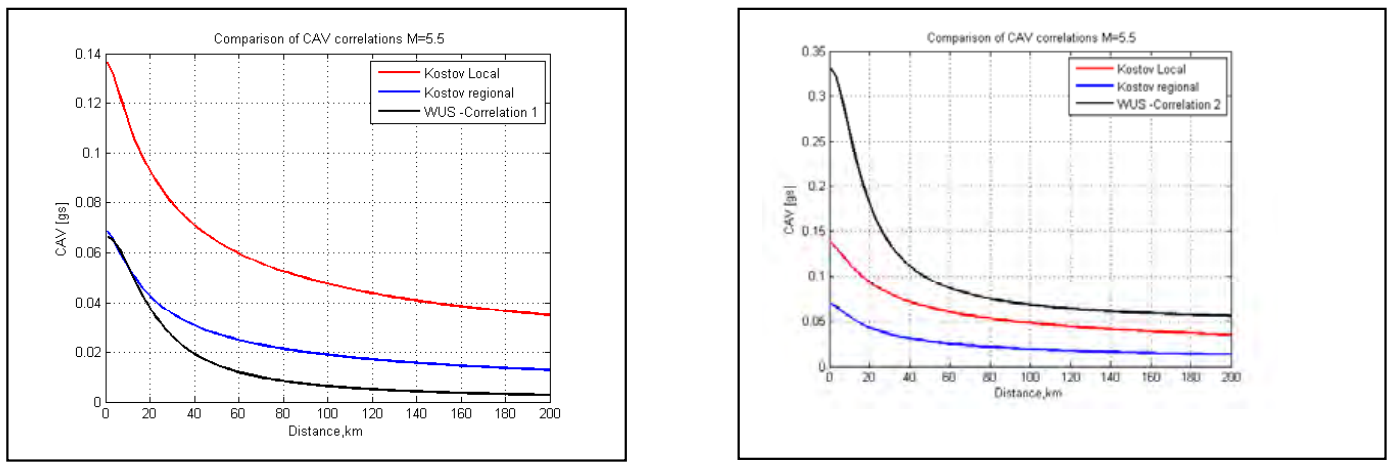

Figure 2. Comparison of Kostov's equations with WUS-correlation 1 and 2 for magnitude 5.5

The obtained equations were used to calculate the probability of exceedance for the damaging threshold of earthquakes as defined in NRC RG 1.166 (CAV > 0.16 gs). For the WUS correlation 2, an additional intermediate step is required to consider the reduction of the used datasets by eliminating short strong motion records (below 1s) statistically. Therefore, the procedure for calculating the exceedance probability of the damaging threshold had to be adjusted by weighting the exceedance of CAV $>0.16$ gs with the probability that a uniform duration larger than 1 s is observed.

$$
P_{D L}=P_{C A V>0.16 g s} P_{d u \geq 1 s}
$$

Here, $P_{D L}$ is the probability of exceedance of the damaging threshold, $P_{C A V>0.16 g s}$ is the probability of exceedance of CAV=0.16 gs obtained by the direct use of WUS-correlation 2 and $P_{d u \geq 1 s}$. Simple linear approximations in dependence of magnitude were obtained for the different distance ranges D1, D2, D3.

\section{DEVELOPMENT OF THE EPISTEMIC UNCERTAINTY DISTRIBUTION FOR THE PROBABILITY OF EXCEEDANCE OF THE DAMAGING THRESHOLD OF EARTHQUAKES}

The exceedance probability of the damaging threshold of earthquakes was calculated for each of the developed equations, according to the bootstrap procedure discussed in section 2. Because the relative performance of the different 
equations may change in dependence of magnitude and distance (see for example figures 3 and 4), the epistemic uncertainty distribution was derived in the form of a lognormal distribution assigning the calculated maximal exceedance probability of the damaging threshold for each of the hazard probabilities (abbreviation for exceedance probability of a given hazard level) to the $95 \%$ quantile of the lognormal distribution and the minimal value to the $5 \%$ quantile. This leads to a conservative stretching of the resulting epistemic uncertainty distribution towards the upper and lower tails increasing the total amount of uncertainty considered in the model. Interpolation between the calculated values of the exceedance probability of the damaging threshold for the different hazard probabilities (which were considered in the hazard disaggregation) was performed using Hermit polynoms. This procedure was used to develop epistemic uncertainty distributions for each of the considered distance ranges (D1, D2, D3). On the basis of the developed distributions a composite uncertainty distribution for the uniform hazard spectrum obtained from the PEGASOS results was developed by weighting the distributions for the separate distance ranges with their relative contribution to the total hazard:

$$
w_{i}(H, f)=\frac{P_{U H S, i}\left(a_{U H S, t o t} \mid(H, f)\right)}{H}
$$

where $w_{i}(H, f)$ is the weight for the distance range $i$ associated with the hazard probability $H$ (e. g. for $10^{-4} / a$ ) and the spectral acceleration $f$, and $P_{U H S, i}\left(a_{U H S, t o t} \mid(H, f)\right)$ is the exceedance probability of the spectral acceleration $a$, corresponding to the hazard probability $H$ and the spectral frequency $f$. Figure 7 shows an example for the resulting epistemic uncertainty distribution for distance range D1 (near site sources), while figure 8 shows the resulting composite epistemic uncertainty distributions for the exceedance probability of the damaging threshold of earthquakes for different spectral frequencies (mean values) with respect to the UHS.
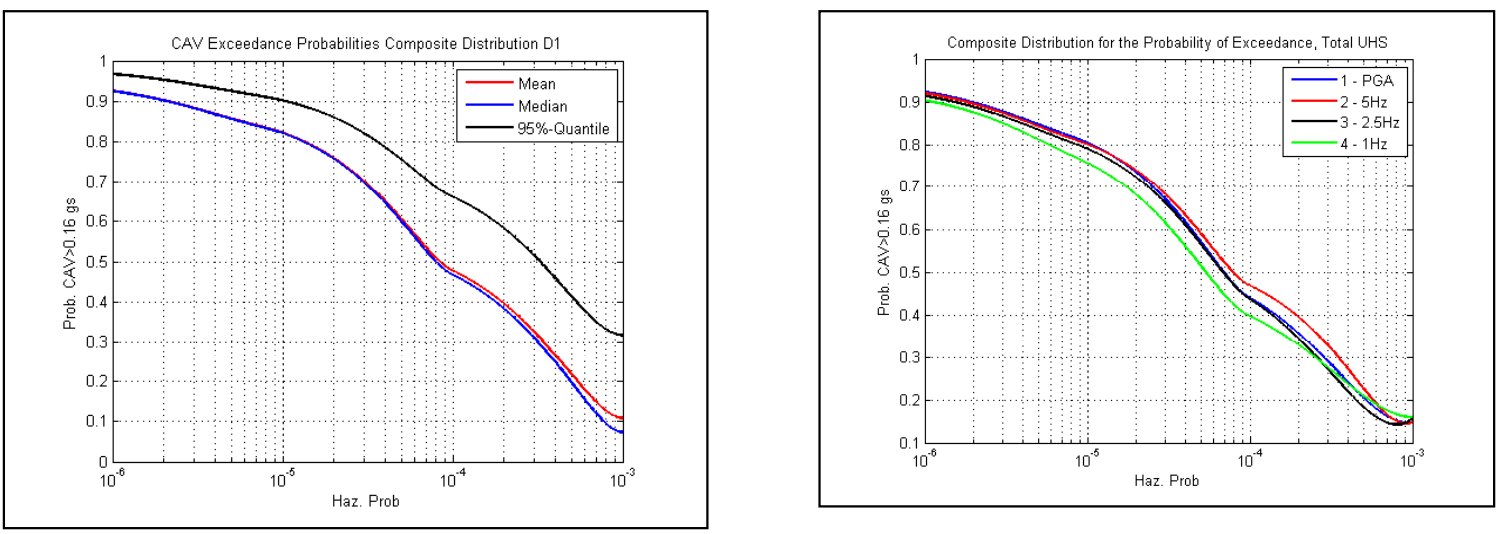

Figure 3. Composite epistemic uncertainty distribution for the probability of exceedance of the damaging threshold for distance range D1 $(<16 \mathrm{~km}, 3$ fractiles) and for the total UHS (frequency dependent, mean)

\section{APPLICATION OF THE PROCEDURE AND CONCLUSIONS}

The developed procedure was implemented into the plant specific seismic PSA. For this purpose an additional top event was incorporated into the seismic event tree questioning the exceedance of the damaging threshold of earthquakes. The first sensitivity analysis performed indicates that a reduction of the mean core damage frequency of about $30 \%$ is to be expected in comparison to a model using the untruncated hazard. The procedure was also applied to compare the seismic design basis of NPP Goesgen (safe shutdown earthquake), which was developed based on a deterministic seismic hazard analysis. Additionally, the results of recent comprehensive geotechnical investigations [20] were incorporated into the analysis. These investigations, which have been validated by a comparison to the large set of borehole information available for the site, have shown that the shear wave velocities of the geological bedrock are significantly lower than the PEGASOS experts expected. The PEGASOS experts in SP3 assumed a hard bedrock layer at a depth of about $30 \mathrm{~m}$ with a shear wave velocity of $2400 \mathrm{~m} / \mathrm{s}$, while the measured values at this depth are around $1100 \mathrm{~m} / \mathrm{s}$. This significantly changes the impedance contrast. 


\section{S-wave velocity contour map for $z=32 \mathrm{~m}$}

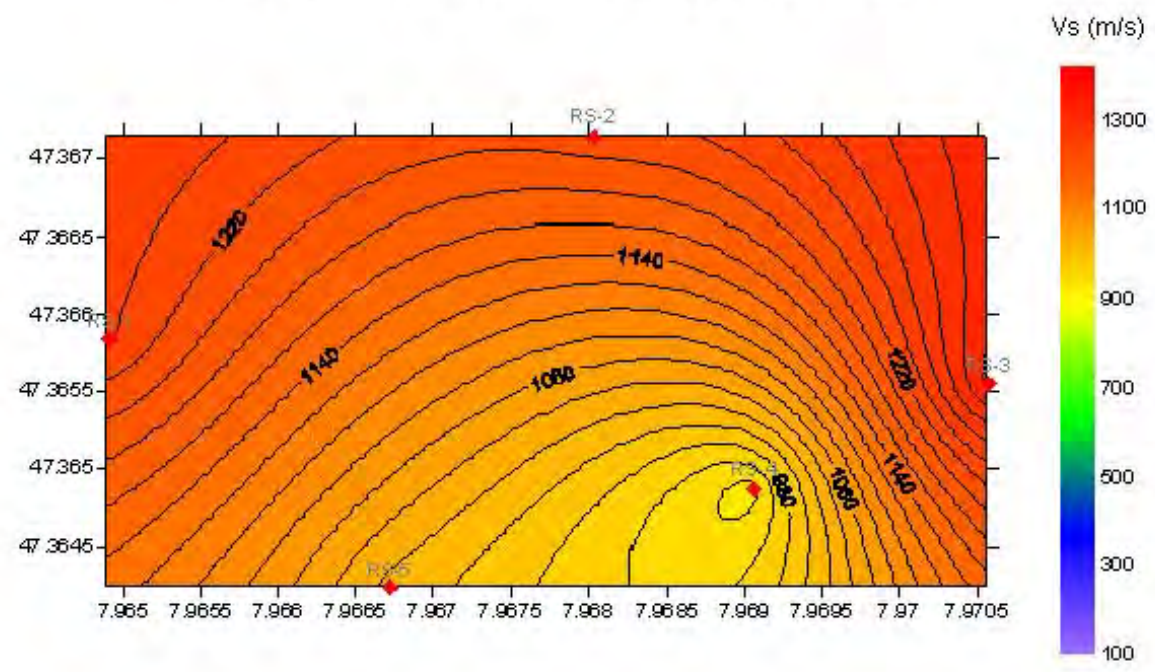

Figure 4. Measured shear wave velocity profile at a depth of $32 \mathrm{~m}$

Figure 9 shows a comparison of the design basis of Goesgen (old SSE, and the "effective" SSE used for new constructions) with a theoretical SSE derived from PEGASOS according to the NRC RG 1.165 procedures (with the exception that the SSE is based on the mean for the exceedance probability of $10^{-4} /$ a according to current Swiss practice and not on the median for $10^{-5} / \mathrm{a}$ ).

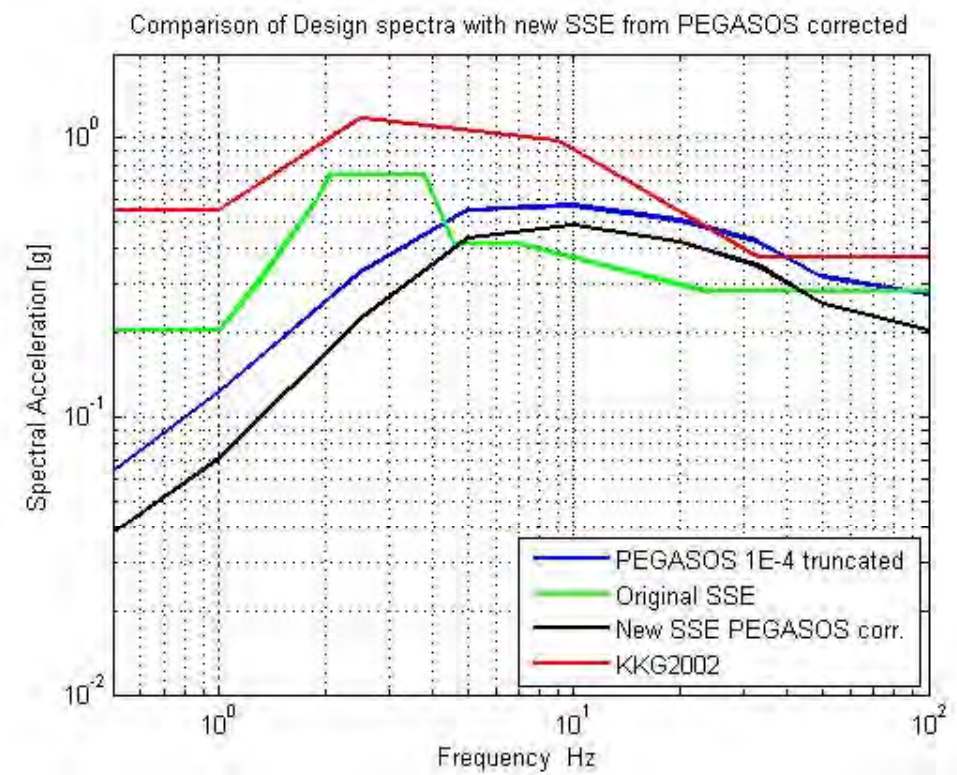

Figure 5. Comparison of deterministic SSE design spectra with the truncated and corrected (true site conditions) PEGASOS SSE

The comparison shows that the deterministic original design basis is in reasonable agreement with a theoretical new SSE derived from PEGASOS, while the revised design basis introduced for new projects in 2002 (KKG 2002) is very conservative. It can be concluded that the use of a damaging threshold in the application of PSHA for risk analysis and design helps to restore energy conservation principles and leads to a more realistic design basis, which is in better compliance with historical observations with respect to damage caused by earthquakes [2]. 


\section{REFERENCES}

1. Senior Seismic Hazard Analysis Committee (SSHAC), 1997. Recommendations for Probabilistic Seismic Hazard Analysis: Guidance on Uncertainty and Use of Experts. NU-REG/CR-6372.

2. Klügel, J.-U., 2005. Problems in the application of the SSHAC probability method for assessing earthquake hazards at Swiss nuclear power plants. Engineering Geology, 78, pp. 285-307.

3. Klügel, J.-U., 2005. Reply to the comment on J.-U. Klügel's "Problems in the application of the SSHAC probability method for assessing earthquake hazards at Swiss nuclear power plants“, in Engineering Geology, Vol. 78, pp. 285307, by Budnitz, by J.-U. Klügel, Engineering Geology, 82, pp. 79-85.

4. Klügel, J.-U., 2005. Reply to the comment on J.-U. Klügel's "Problems in the application of the SSHAC probability method for assessing earthquake hazards at Swiss nuclear power plants“, in Engineering Geology, Vol. 78, pp. 285307, by Musson, Engineering Geology, 82, pp. 56-65.

5. Klügel, J.-U., 2007. Error inflation in Probabilistic Seismic Hazard Analysis, Engineering Geology, 90, pp. $186-192$.

6. Cooke, R.M., 1991. Experts in Uncertainty. Opinion and subjective probability in science. Oxford University Press., Oxford.

7. Cooke, R.M., Expert Judgement in Seismic Risk: Summary and Recommendations Based on Performance Based Experts Aggregation by Chunfang Meng, 2005

8. Vanmarcke, E.H. and Lai, S.S.P., 1980. Strong Motion Duration and RMS Amplitude of Earthquake Records, Bulletin of the Seismological Society of America, Vol. 70, No. 4, pp.1293-1307.

9. Arias, A., 1970. A measure of earthquake intensity. In R.J. Hansen (Eds.): Seismic Design for Nuclear Power Plants, MIT Press, Cambridge, Massachusetts.

10. Bertero, V.V. and Uang, C.M., 1992. Issues and future directions in the use of an energy approach for seismicresistant design of structures, In nonlinear seismic analysis and design of reinforced concrete buildings, Ed. P. Fajfar and H. Krawinkler, 3-22.

11. NRC RG 1.166

12. Miller, A.C. and Rice, T.R., 1983. Discrete approximations of probability distributions. Management Science, 29, pp. 352-362.

13. NRC RG 1.165

14. Bazzuro, P. and Cornell, C.A., 1999. Disaggregation of Seismic Hazard, Bulletin of the Seismological Society of America, 82, pp. 501-520.

15. Kostov, M. K., 2005. Site Specific Estimation of Cumulative Absolute Velocity, in Proceedings of 18th International Conference on Structural Mechanics in Reactor Technology, Beijing, August 7 - 12, 2005.

16. Ekström, G. and Dziewonski, A.M., 1988. Evidence of bias in estimation of earthquake size. Nature, 332, pp.319323.

17. Toro, G.R., 2003. Technical Note to SP1 experts on the treatment of Hypocentral Depths and Rupture-Length Effects for Area Sources in the FRISK88MP Software, 19.5.2003.

18. EPRI, Program on Technology Innovation: Use of CAV in Determining Effects of Small Magnitude Earthquakes on Seismic Hazard Analyses, TI-1012965, Dec 2005.

19. Bolt, B.A.,1973. Duration of Strong Ground Motion, Proc. Fifth World Conf. Earth Eng., Rome, 1304-1307.

20. Anatolian Geophysical, 2006. Report on Shallow Seismic Investigation at NPP Gösgen Site, Switzerland. 